Planetary Systems in the Universe - Observation, Formation and Evolution

Proceedings IAU Symposium No. 202, (C2004 IAU

Alan Penny, Pawel Artymowicz, Anne-Marie Lagrange, \& Sara Russell, eds.

\title{
The inner 1 AU of circumstellar disks
}

\author{
R.L. Akeson ${ }^{1}$, D.R. Ciardi ${ }^{2}$, G.T. van Belle ${ }^{3}$, M.J. Creech-Eakman ${ }^{3}$, \\ and E.A. Lada ${ }^{2}$ \\ ${ }^{1}$ IPAC/California Institute of Technology, MS 100-22, Pasadena, \\ CA 91125, USA \\ ${ }^{2}$ University of Florida, Gainesville, FL 32611, USA \\ ${ }^{3}$ Jet Propulsion Laboratory, California Institute of Technology, \\ MS 171-113, 4800 Oak Grove, Pasadena, CA 91109, USA
}

\begin{abstract}
Infrared observations of two T Tauri stars, T Tau and SU Aur, were obtained using long-baseline interferometry with the Palomar Testbed Interferometer (PTI). For both sources, the 2.2 micron emission is resolved at PTI's nominal fringe spacing of 4 milliarcsec. Using simple circumstellar material distributions, we find that the infrared emission region is tenths of AU in size. We also compare our results from PTI with accretion disk models inferred from spectral energy distributions.
\end{abstract}

\section{Introduction}

The structure of young stellar object (YSO) circumstellar disks has been studied using spectral energy distributions (SED), spectral line profiles and imaging at infrared and (sub)-millimeter wavelengths. The dust continuum emission from disks around several $\mathrm{T}$ Tauri sources has been resolved at millimeter wavelengths. These observations are sensitive to emission from cooler dust and provide spatial information on size scales of several 10's of AU. The disk physical properties on much smaller scales $(<$ few $\mathrm{AU})$ are generally inferred through examination of the spectral line shapes and modeling of the SED. Infrared interferometry provides a method to directly observe the inner disk. To date, only a few YSOs have been observed using this technique (e.g. FU Ori: Malbet et al. 1998 and AB Aur: Milan-Gabet et al 1999). Here we present K-band (2.2 $\mu \mathrm{m})$ long baseline interferometric observations of two T Tauri stars, T Tau and SU Aur. These results, along with observations of two other YSOs (MWC 147 and AB Aur) are detailed in Akeson et al. 2000.

\section{Observations}

The data were taken at the Palomar Testbed Interferometer (Colavita et al. 1999) between September and December 1999. The data were calibrated using the standard method described in Boden et al. 1998. The system visibility, the visibility of an unresolved object, is measured using calibrator stars. Calibrators were chosen for their proximity to the source and for small angular size, minimizing systematic errors in deriving the system visibility. The data are pre- 
sented in normalized squared visibility $\left(\mathrm{V}^{2}=1\right.$ for an unresolved source). The uncertainties for the calibrated visibilities are a combination of the calibrator size uncertainty and the internal scatter in the data.

\section{Results}

We fit simple geometric models, uniform and Gaussian disks, to the data as size scale estimators. We also compare the data to accretion disk models from the literature. For a face-on Gaussian or uniform profile, the predicted visibility is simply a function of the projected baseline. For an inclined profile, the visibility is also a function of hour angle. As neither source shows definitive visibility structure with hour angle, we limit ourselves to the simple face-on case.

Emission from an accretion disk is one of the leading explanations for the infrared excess and other observed features of $\mathrm{T}$ Tauri stars. As we have only sampled one spatial scale in the disk, we will use accretion disk models from the literature, where the SED or millimeter imaging has been used to determine the disk parameters.

\subsection{T Tau}

$\mathrm{T}$ Tauri, one of the best-studied YSOs, has an infrared companion, T Tau S, 0 '.7 to the south, which is optically obscured. Both components have a nearinfrared excess, suggestive of circumstellar material. At $\mathrm{K}$ band, $\mathrm{T}$ Tau $\mathrm{N}$ is the component with higher flux and is the source of the measured fringes (see further discussion in Akeson et al. 2000). The data were corrected for the effects of $\mathrm{T}$ Tau $\mathrm{S}$ before fitting.

The data were reduced and binned by projected baseline before being fit by Gaussian and uniform disk models (Figure 1). The stellar photosphere contribution is included as a point source. For the accretion disk model, we have used the parameters derived by Ghez et al. (1991) and Akeson et al. (1998). These accretion disk models overestimate the measured visibility and, thus, underestimate the size scale of the $\mathrm{K}$ band emission.

\subsection{SU Aur}

SU Aur is a $\mathrm{T}$ Tauri star with an SED similar to that of $\mathrm{T}$ Tau. As for $\mathrm{T}$ Tau, the data were binned by projected baseline before fitting (Figure 1). The accretion disk model is taken from Beckwith et al. (1990), where the parameters were determined by fitting $10 \mu \mathrm{m}$ through millimeter wave fluxes. This model overestimates the observed visibility.

\section{Discussion}

The fundamental result of these observations is that for both $\mathrm{T}$ Tauri sources presented here, the infrared emission arising from circumstellar material is resolved by PTI with a nominal fringe spacing of 4 mas. The measured sizes correspond to physical scales of tenths of AU. Our data on T Tau and SU Aur require the $\mathrm{K}$ band emission to come from a larger region than that predicted by the accretion disk models. 

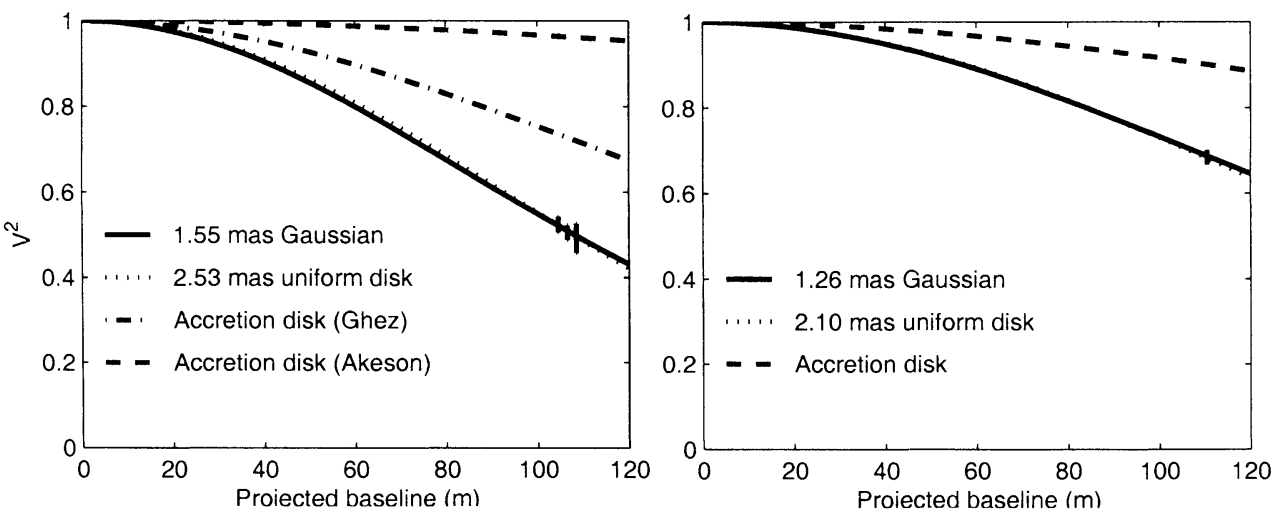

Figure 1. PTI data points, binned by projected baseline length, and models for T Tau (left) and SU Aur (right).

Acknowledgments. This work was performed at the Infrared Processing and Analysis Center, California Institute of Technology and the Jet Propulsion Laboratory. Data were obtained at the Palomar Observatory using the NASA Palomar Testbed Interferometer, which is supported by NASA contracts to the Jet Propulsion Laboratory. Science operations with PTI are possible through the efforts of the PTI Collaboration ( http://huey.jpl.nasa.gov/palomar/ ptimembers.html ). We particularly thank A. Boden for his efforts in data reduction software and B. Thompson for useful discussions. We are also grateful to $\mathrm{T}$. Beck for providing the $\mathrm{T}$ Tau flux ratio.

\section{References}

Akeson, R.L., Ciardi, D.R., van Belle, G.T., Creech-Eakman, M.J., and Lada, E.A. $2000, \mathrm{ApJ}$, to appear in the Nov. 1 issue

Akeson, R. L., Koerner, D. W., and Jensen, E. L. N. 1998, ApJ, 505, 358

Beckwith, S. V. W., Sargent, A. I., Chini, R. S., and Gusten, R. 1990, AJ, 99, 924

Boden, A. F., Colavita, M. M., van Belle, G. T., and Shao, M. 1998, SPIE proceedings, 3350,872

Colavita M. M. et al. 1999, ApJ, 510, 505

Ghez, A. M., Neugebauer, G., Gorham, P. W., Haniff, C. A., Kulkarni, S. R., Matthews, K., Koresko, C., and Beckwith, S. 1991, AJ, 102, 2066

Malbet, F., et al. 1998, ApJ, 507, 149

Millan-Gabet, R., Schloerb, F. P., Traub, W. A., Malbet, F., Berger, J. P., and Bregman, J. D. 1999, ApJ, 513, 131 